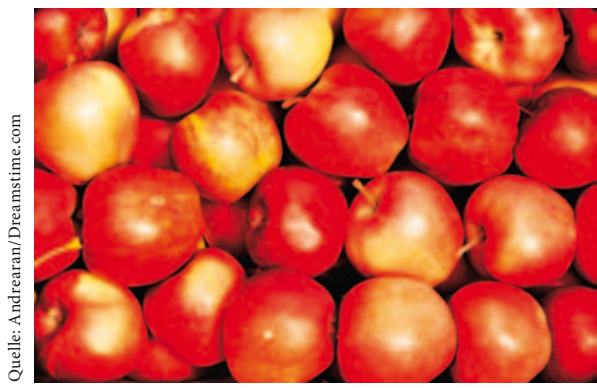

Am besten viel Obst, Gemüse und Ballaststoffe, so kann man den Trend in der modernen Ernährungslehre zusammenfassen. An Schweizer Schulen finden jedes Jahr Apfelwochen statt, in denen Pausenäpfel verteilt werden, die Grossverteiler werben für den Konsum von Rohkost und die Schweizer Krebsliga hat die Kampagne «5 am Tag» lanciert. Diese Aktion ist Teil einer seit über zehn Jahren international laufenden Gesundheitsaktion, die zum Ziel hat, durch den gesteigerten und regelmässigeren Konsum von Obst und Gemüse einen Beitrag zur Krebsprävention zu leisten. Fünfmal am Tag soll man verschiedene Rohkostgerichte zu sich nehmen, um sich vor Krebs zu schützen. Der World Health Report schreibt den Tod von jährlich 2,7 Millionen Menschen einer zu geringen Aufnahme von Obst und Gemüse zu. Vom Rohkostmangel sind die meisten Länder betroffen. Die Experten der WHO gehen davon aus, dass die Förderung des Obst- und Gemüsekonsums hilft, chronische Krankheiten zu verhindern, Mangelerscheinungen vorzubeugen und die Widerstandsfähigkeit gegenüber Infektionskrankheiten zu erhöhen.

Andere Experten sind allerdings skeptisch, ob die internationale Kampagne «5 am Tag» tatsächlich die gewünschte positive Wirkung auf die Krebsprävention hat. «Die ursprüngliche Idee, mit «5 am Tag〉 Krebsprävention zu betreiben, lässt sich wissenschaftlich nicht halten», sagt Paolo Colombani, der an der ETH Zürich

\title{
Obst und Gemüse schützen vor Herzinfarkt
}

Die Kampagne «5 am Tag» propagiert den Konsum von Obst und Gemüse. Mindestens fünfmal täglich soll man zum Beispiel Äpfel, Bananen oder Salat essen. Ob die Rohkost vor Krebs schützt, ist unklar. Die Hinweise, dass jede Obstportion das Herzinfarktrisiko um 10\% senkt, sind deutlich.

über die Ernährungsgewohnheiten der Bevölkerung forscht. «Ich finde die Kampagne nicht schlecht, weil Gemüse und Früchte helfen, Übergewicht zu vermeiden.» Geändert habe sich in den letzten Jahren allerdings die Begründung, sie durchzuführen. Die Krebsprävention steht nicht mehr im Mittelpunkt. Stattdessen wird die Bedeutung von Obst und Gemüse zur Vermeidung anderer Krankheiten stärker betont.

\section{Studien belegen Schutzwirkung}

Was eine ganze Reihe von Erkrankungen betrifft, gibt es tatsächlich Studien, die die Schutzwirkung von Obst- und Gemüsekonsum belegen. Das gilt insbesondere für die Prävention von Herz-Kreislauf-Erkrankungen. In der Multicenter-Studie CARDIO2000, deren Ergebnisse 2003 im Nutrition Journal veröffentlicht wurden, fanden die Autoren einen direkten Zusammenhang zwischen der Anzahl der Frucht- und Obstmahlzeiten und dem Risiko ihrer Testpersonen, an einer Herz-Kreislauf-Krankheit zu erkranken. Jede Portion Obst und Gemüse reduzierte die Wahrscheinlichkeit, einen Herzinfarkt zu erleiden, um $10 \%$. Diejenigen, die am meisten von diesen Naturprodukten assen, hatten ein um $72 \%$ verringertes Risiko gegenüber denjenigen, die am wenigsten davon assen: Wer mehr als fünfmal pro Tag Obst und Gemüse zu sich nahm, war somit deutlich besser geschützt als jemand, bei dem das nicht auf dem täglichen Speisezettel stand. Wer drei- mal in der Woche Obst oder Gemüse ass, hatte im Vergleich zu den Abstinenten ein um 70\% reduziertes Risiko. Für die Autoren sind diese Ergebnisse ein ausreichender Grund, eine Aufklärung der Bevölkerung über die gesunde Ernährung zu fordern, um den Konsum von Obst und Gemüse zu steigern. Allerdings warnen sie, dass die Resultate ihrer Studien noch kein Beleg für eine Kausalität seien.

\section{Positiver Effekt auf das}

Herz-Kreislauf-System

Auch neuere Untersuchungen deuten darauf hin, dass ein höherer Konsum von Obst und Gemüse eine Wirkung auf die Häufigkeit von Herz-Kreislauf-Erkrankungen hat. In einer Studie, die im Februar 2010 im British Journal of Nutrition veröffentlicht wurde, wurde besonders die Schutzwirkung von Zitrusfrüchten, Äpfeln und Kohlgemüse bei knapp 1100 Männern und Frauen zwischen 50 und 64 Jahren, die einen Herzanfall erlitten hatten, untersucht. Bei Männern und Frauen mit einem hohen Obstund Gemüsekonsum war das Risiko eines Infarkts geringer. Allerdings fanden die Autoren, dass diejenigen Männer, die viele Äpfel assen, ein erhöhtes Risiko für einen Infarkt hatten. Bei Frauen fanden sie die gleiche Tendenz; sie war allerdings knapp nicht signifikant. Frauen, die grössere Mengen an Fruchtsäften tranken, hatten ebenfalls ein höheres Risiko. (rfi)

\section{KARGER}

Fax +497614520714 